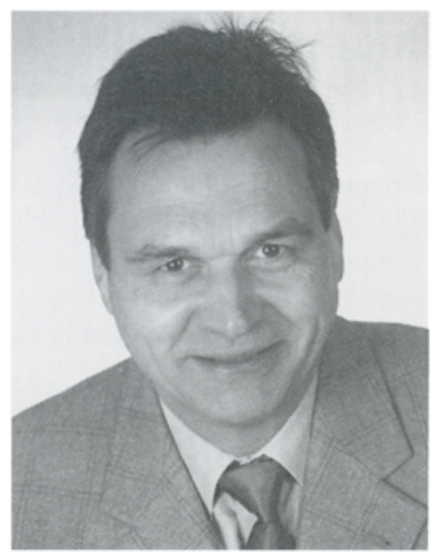

Uwe Fichtner

\section{Zeiten der Trauer und der Besinnung}

Der Herbst des Jahres 2001 hat der Welt Terror, tausendfachen Tod und Krieg gebracht. Über derartige globale Ereignisse wortlos hinwegzugehen, stünde einer Vereinigung, die sich der Förderung der Kartographie verpflichtet sieht, gewiss nicht gut an. Neben zahlreichen anderen Gründen wäre es auch aus wissenschaftsethischen Aspekten durchaus angebracht, sich in dieser Zeit zu vergegenwärtigen, wie sehr unser Fachgebiet und die aus ihm entwickelten Techniken einerseits im Dienste von Wissenschaft und Gesellschaft stehen, anderseits aber eben auch für inhumane Zwecke instrumentalisiert wurden und instrumentalisiert werden können. Zu diesem Spannungsfeld haben sich Fachvertreter bislang relativ wenig geäußert - vielleicht böte eines der kommenden Symposien zur Theoretischen Kartographie einen geeigneten Rahmen, um diese Thematik aufzugreifen und intensiver zu beleuchten.

In diesem Herbst starb Dr. Klaus Kirschbaum, unser Verleger. Die Deutsche Gesellschaft für Kartographie beklagt seinen Tod und kondoliert der Familie. Mit inm verlor die Kartographie einen großen Förderer. Den Nachruf auf diese außergewöhnliche Persönlichkeit hat dankenswerter Weise J. Dodt verfasst Is. in diesem Heftl. Alle, die den 50. Deutschen Kartographentag in Berchtesgaden besucht haben - und es waren leider viel zu wenige - werden wohl zustimmen, dass man dem gesamten örtlich vorbereitenden Ausschuss nicht nachdrücklich genug für die hervorragende Organisation und die viele Mühe danken kann. Unter schwierigen Voraussetzungen hat er mit einer relativ kleinen Zahl von Mitarbeitern und trotz verhältnismäßig geringer Unterstützung von Seiten der behördlichen Kartographie einen Kongress "auf die Beine" gestellt, der sich wirklich sehen lassen konnte und der bei den Teilnehmern auf ein sehr positives Echo stieß, wie sich in den Gesprächen zeigte. Im Nachklang zum Kartographentag muss man auch ohne Zweifel konsta- tieren, dass sich alle Anwesenden in den Diskussionen bemühten, einen passablen Weg für einen Neuanfang aus der schwierigen Situation, die in der Neuwahl des Vorstandes bestand, zu finden. Dass dies gelungen ist, macht Mut für die Zukunft. Der neugewählte Vorstand hat sich sofort mächtig "ins Zeug gelegt", wie man seiner in diesem Heft abgedruckten ersten Verlautbarung, den Protokollen und den Berichten aus den Gremiensitzungen entnehmen kann.

Das vorliegende Heft zeigt am Beispie von zwei besonders innovativen Gebieten, dem Einsatz never Satellitensensoren und der Datenvisualisierung im Internet, welche Chancen und Probleme die jüngsten technischen Entwicklungen bieten. Den Blick zurück auf die Vergangenheit werfen zwei weitere Beiträge, in denen interdisziplinäre Beziehungen unseres Fachgebietes zur Geographie, vielfältige historische Bezüge sowie die Bedeutung des Standortes Leipzig für die deutsche Kartographie und der technische Wandel in der Kartenredaktion zum Ausdruck kommen.

Wieder einmal verdient die Titelseite der $\mathrm{KN}$ besondere Aufmerksamkeit und Beachtung: sie gibt einen Ausschnitt aus dem ersten Kartenblatt des neven zivil-militärisch topographischen Kartenwerks im Maßstab 1:50000 wieder. Dieses selbstverständlich sowohl in analoger wie in digitaler form hergestellte Produkt dürfte in Zukunft weite Verbreitung finden, ist doch die Bundeswehr Hauptabnehmer dieses Kartenwerkes, das damit zugleich auch im Rahmen der Nato eingesetz $\dagger$ werden wird.

Zum Jahreswechsel dankt Ihnen, liebe Leserinnen und Leser, die Redaktion für Ihr beständiges Interesse an den KN und wünscht uns allen ein im wahrsten Sinne des Wortes friedliches Weihnachtsfest,

Ihr 